$12-2005$

\title{
Effect of Gender on Lower Extremity Kinematics During Rapid Direction Changes: An Integrated Analysis of Three Sports Movements
}

S. G. McLean

Cleveland Clinic Foundation, mcleans@ccf.org

K. B. Walker

Cleveland Clinic Foundation

Antonie J. van den Bogert

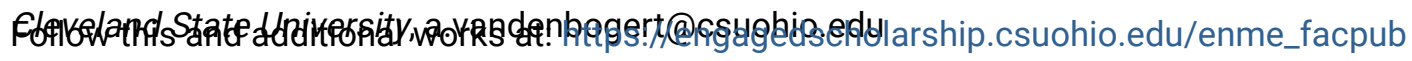

Part of the Biomechanical Engineering Commons

How does access to this work benefit you? Let us know!

\section{Publisher's Statement}

NOTICE: this is the author's version of a work that was accepted for publication in Journal of Science and Medicine in Sport. Changes resulting from the publishing process, such as peer review, editing, corrections, structural formatting, and other quality control mechanisms may not be reflected in this document. Changes may have been made to this work since it was submitted for publication. A definitive version was subsequently published in Journal of Science and Medicine in Sport, 8, 4, (12-01-2005); 10.1016/S1440-2440(05)80056-8

\section{Original Citation}

McLean, S. G., Walker, K. B., and van den Bogert, A., 2005, "Effect of Gender on Lower Extremity Kinematics during Rapid Direction Changes: An Integrated Analysis of Three Sports Movements," Journal of Science and Medicine in Sport, 8(4) pp. 411-422.

This Article is brought to you for free and open access by the Mechanical Engineering Department at EngagedScholarship@CSU. It has been accepted for inclusion in Mechanical Engineering Faculty Publications by an authorized administrator of EngagedScholarship@CSU. For more information, please contact library.es@csuohio.edu. 


\title{
Effect of gender on lower extremity kinematics during rapid direction changes: an integrated analysis of three sports movements
}

\author{
SG McLean, KB Walker \& AJ van den Bogert \\ The Cleveland Clinic Foundation, Cleveland, Ohio, USA.
}

\section{Introduction}

Anterior cruciate ligament (ACL) injury is a common and potentially traumatic knee joint injury. Approximately 100000 ACL injuries occur annually within the United States ${ }^{1}$, with the majority of these suffered by young healthy individuals. Surgical reconstruction is often necessary, followed by a difficult and lengthy rehabilitation program ${ }^{2}$. ACL injury also predisposes the patient to significant long-term consequences such as osteoarthritis ${ }^{3}$. Of particular concern is the significant gender disparity in non-contact ACL injury rates. In sports such as basketball, soccer and team handball, for example, women are reported to suffer ACL injuries 2-7 times more often than men ${ }^{1.4}$. With this in mind, elucidating the causal factors of ACL injury that are amenable to intervention is paramount, particularly for females. 
Altered neuromuscular control in females during movements incorporating rapid changes in speed or direction is suggested to increase their risk of ACL injury compared to males ${ }^{4,5}$. Neuromuscular intervention strategies have thus evolved, aimed at preventing female ACL injuries via modification of what are considered "high-risk" movement patterns ${ }^{5,6}$. Recent data indeed suggest that modification of lower limb movement control is possible ${ }^{6,7}$. The ultimate success of these programs, however, is reliant on accurate determination of which patterns are in fact high-risk.

Neuromuscular predictors of sports-related ACL injuries in women have typically been proposed based on gender comparisons of joint motions during isolated sports movements only $8,9,10$. Such studies have afforded greater insight into potential causal factors of ACL injury for each of these movements. It remains unclear, however, as to whether or not neuromuscular predictors of ACL injury are transferable across movements. If these predictors are largely task-specific, then the efficacy of current ACL injury screening protocols, which are typically based on a single movement ${ }^{5,6}$, will be severely compromised. Similarly, neuromuscular training programs typically target a specific movement, such as jump landing, and it is not yet known whether or not training effects will transfer to other movements linked to ACL injury.

Previous research into gender-specific neuromuscular control and ACL injury risk has also focused almost exclusively on the knee joint ${ }^{8,9,11}$. It is increasingly recognised, however, that "high-risk" knee biomechanics may potentiate through altered neuromuscular control elsewhere in the lower extremity ${ }^{10,12}$. Recent studies have begun to address this concern by extending movement analyses beyond the knee joint, but have typically done so for low-risk movements in which ACL injury is either unlikely ${ }^{13,14}$, or have limited evaluation to the sagittal plane ${ }^{15,16}$.

Considering the above research shortcomings, gender specific 3D kinematic characterisations for the entire lower limb across movements in a single population appear necessary. These data would provide further insight into the potential gender-specificity in causal factors linked to ACL injury, and would facilitate more effective screening of individuals at increased risk of injury due to their demonstrated neuromuscular control. The purpose of the current study, therefore, was to examine gender differences in 3-D hip, knee and ankle kinematics during the stance phase of three previously established high-risk sporting movements; namely, jump-landing, sidestepping and shuttle run tasks. The extent to which observed differences were consistent across movements was subsequently examined.

\section{Materials and Methods Subjects}

Our previous research comparing male and female knee joint (rotational) kinematic data for sidestepping tasks found that the ratio of within-group to between-group differences was $0.73^{17}$. The current study made statistical comparisons of 24 hip (9), knee (9) and ankle (6) dependent measures, between both genders (2) and movements (3). A power analysis revealed that to achieve $80 \%$ statistical power in the current study with an exploratory alpha level of 0.05 , a minimum of eight subjects per group (male and female) were required. Ten female (height $=176.0 \pm 11.1 \mathrm{~cm}$, weight $=76.1 \pm 12.4 \mathrm{~kg}$, age $=21.1 \pm 3.0 \mathrm{y}$ ) 
and 10 male (height $=184.7 \pm 8.0 \mathrm{~cm}$, weight $=81.9 \pm 9.8 \mathrm{~kg}$, age $=20.2 \pm 1.9 \mathrm{y}$ ) NCAA Division 1 basketball players were thus recruited for the study. Subjects were also matched across gender for experience (female $=10.5 \pm 4.8 \mathrm{y}$, male $=10.2$ ( $5.1 \mathrm{y}$ ), being denoted by the number of years participating in organised basketball. Prior to testing, research approval through the Institutional Review Board of the Cleveland Clinic Foundation and written informed consent for all subjects was obtained. Subject inclusion was based on no history of operable lower limb joint injury and a proficiency in performing the sporting movements under investigation.

\section{Methodology}

For each subject, hip, knee and ankle joint 3D kinematics were recorded for the right (contact) leg during the execution of three sports-specific movements; namely jump-landing, sidestepping and shuttle running. Subjects performed 10 successful trials for each of the three movement conditions, which required the contact phase of the movement to occur on a force plate (AMTI OR6-5 \#4048, Advanced Mechanical Technology Inc) within the field of view of a sixcamera high-speed $(240 \mathrm{~Hz})$ motion analysis system (Motion Analysis Corporation, Santa Rosa, CA). Approach speeds for all trials were required to fall between $4.5 \mathrm{~m} . \mathrm{s}^{-1}$ and $5.5 \mathrm{~m} . \mathrm{s}^{-1}{ }^{10}$. These speeds were monitored during testing via a stopwatch over a 3-m distance immediately on the approach side of the force plate, to ensure a minimum of 10 successful trials. Trial speeds were also accurately post-processed as will be described later.

For the sidestep, cutting angles were between $35^{\circ}$ and $55^{\circ}$ from the original direction of motion, with this range demarcated (with tape) by lines on the floor, originating at the centre of the forceplate. Subjects continued running following sidestep execution for approximately five steps, and initial foot contact following the cutting action was required to fall within this prescribed range. For the sidejump, subjects ran and then initiated a jump approximately $2 \mathrm{~m}$ on the approach side of the force plate. Upon landing on the force plate with the right foot, they pushed-off perpendicular (to the left) to their original direction of motion and landed approximately $1.5 \mathrm{~m}$ to the left of the force plate centre. For the shuttle run, subjects initiated a rapid direction change immediately following contact with the force plate, such that their new direction of motion was approximately $180^{\circ}$ to their original path. Similar to the sidestep, subjects continued running for approximately five steps following the manoeuvre.

For each subject, 21 reflective markers of diameter $24 \mathrm{~mm}$ were secured with strapping tape to specific lower limb anatomical locations, similar to that which we have adopted previously ${ }^{18}$. Attachment sites were shaved and attachment over areas of large muscle mass was avoided to minimise excessive marker movement during initial contact. A static trial was first recorded with the subject standing in the neutral position ${ }^{10,18}$, following which the left and right ASIS, medial femoral condyle and medial and lateral malleoli markers were removed.

\section{Kinematic Model}

From the standing trial, a kinematic model was generated by defining five skeletal segments (foot, talus, shank and thigh of the support limb, and the pelvis) and 14 degrees of freedom using Mocap Solver 6.17 (Motion Analysis 
Corp., Santa Rosa, California), as described previously ${ }^{10}$. Specifically, the pelvis was assigned six degrees of freedom (DOF) relative to the global (laboratory) coordinate system, with the hip, knee and ankle joints defined locally and assigned three, three and two rotational DOF, respectively. Joint coordinate axis orientations were such that for the hip joint, flexion, adduction and internal rotation were denoted as positive. At the knee joint, extension, varus and internal rotation were defined as positive, while at the ankle, dorsiflexion and supination were positive ${ }^{10}$.

The 3D marker trajectories recorded during the test trials for each subject were processed by the Mocap Solver software to solve for the 14 DOF of the kinematic model at each time frame. Joint excursions for the hip, knee, and ankle were calculated relative to a neutral (zero) position in which all segment axes were aligned ${ }^{10,19}$. These data were then low-pass filtered with a cubic smoothing spline with an equivalent cut-off frequency of $20 \mathrm{~Hz}^{20}$. Synchronous 3D ground reaction force data were collected during each movement trial at $1000 \mathrm{~Hz}$ with the AMTI forceplate and were used to time-normalise the joint rotation data to $100 \%$ of stance, being resampled at $1 \%$ increments $(\mathrm{N}=101)$. The stance phase was defined as the time interval in which the vertical force exceeded $10 \mathrm{~N}$.

\section{Dependent Measures}

For each trial, initial contact positions of the eight lower limb rotational DOFs were recorded. A peak stance value was also recorded, with the chosen peak (eg, flexion or extension) being that which deviated the most from neutral. For each subject, the between-trial $(n=10)$ variability demonstrated during stance in each DOF for each of the three movements was also calculated. Specifically, the variance in each DOF across the 10 movement trials was determined at each time-step $(n=101)$, from which the mean standard deviation during stance was obtained. The velocity of the greater trochanter marker X-coordinate (original direction of motion) was also measured for the 10 video frames prior to foot contact, from which approach velocity was calculated ${ }^{10}$.

\section{Statistical Treatment}

Individual trial velocity data were submitted to a three-way ANOVA to verify that gender, movement condition or subject did not influence approach velocity, with subject nested within movement condition and treated as a random effect. Mean subject-based dependent measures were submitted to a twoway mixed design ANOVA to test for the main effect of gender and interactions between gender and movement condition, with movement condition treated as a repeated measure in each subject. Subject was again nested within movement condition and treated as a random factor. In instances where significant differences between gender and/or interactions between gender and movement condition effects were observed, Tukey post-hoc analyses determined where they occurred. Being an exploratory rather than a hypothesis-testing study, we chose to test for statistical significance with an alpha level of 0.05. Correlations were quantified between movement tasks for peak knee valgus measures, by computing the Pearson product moment correlation coefficient ( $r$ ) for the mean subject data values $(\mathrm{N}=20)$. Isolated evaluation of valgus data was based on the fact that it has recently been shown to be the primary prospective predictor of non-contact ACL injury risk in females ${ }^{21}$. 


\section{Results}

Approach velocities were similar between male $\left(4.84 \pm 0.21 \mathrm{~m} . \mathrm{s}^{-1}\right)$ and female $\left(4.86 \pm 0.24 \mathrm{~m} . \mathrm{s}^{-1}\right)$ (observed power $\left.=0.997\right)$ and between sidestep $(4.85 \pm 0.17$ $\left.\mathrm{m} . \mathrm{s}^{-1}\right)$, sidejump $\left(4.81 \pm 0.26 \mathrm{~m} . \mathrm{s}^{-1}\right)$ and shuttle run $\left(4.88 \pm 0.31 \mathrm{~m} . \mathrm{s}^{-1}\right)$ (observed power $=0.96$ ) conditions. Subject was not observed to have a statistically significant main or interactive effect on these comparisons. The remaining statistical comparisons were thus deemed not influenced by approach speed.

\begin{tabular}{lccccccc}
\hline & \multicolumn{2}{c}{ Sidestep } & \multicolumn{2}{c}{ Jump-Land } & \multicolumn{2}{c}{ Shuttle Run } \\
Rotation ${ }^{\xi}$ (degrees) & Male & Female & Male & Female & \multicolumn{2}{c}{ Male } & Female \\
\hline Hip Flexion* & $49.5 \pm 8.1$ & $45.0 \pm 8.8$ & $53.1 \pm 12.1$ & $48.8 \pm 7.2$ & $54.7 \pm 12.2$ & $40.3 \pm 10.1^{\xi}$ \\
Hip Abduction & $-24.3 \pm 6.7$ & $-27.4 \pm 5.1$ & $-29.5 \pm 4.3$ & $-30.1 \pm 4.7$ & $-26.0 \pm 6.0$ & $-27.8 \pm 4.1$ \\
Hip Int Rotation & $19.3 \pm 8.3$ & $22.0 \pm 8.7$ & $17.1 \pm 8.1$ & $21.6 \pm 9.7$ & $24.1 \pm 8.0$ & $20.8 \pm 7.7^{\xi}$ \\
Knee Flexion* & $-65.3 \pm 5.7$ & $-61.9 \pm 5.7$ & $-65.8 \pm 9.9$ & $-63.5 \pm 5.3$ & $-71.5 \pm 3.4$ & $-66.3 \pm 3.6$ \\
Knee Valgus* & $-3.8 \pm 3.9$ & $-10.6 \pm 4.1^{*}$ & $-2.7 \pm 3.9$ & $-9.2 \pm 3.9^{\xi}$ & $-5.6 \pm 2.4$ & $-11.1 \pm 3.5^{\xi}$ \\
Knee int Rotation & $19.0 \pm 5.3$ & $17.7 \pm 8.6$ & $19.1 \pm 4.8$ & $19.8 \pm 8.1$ & $10.0 \pm 8.3$ & $14.7 \pm 10.2$ \\
Ankle Dorsiflexion & $4.9 \pm 5.8$ & $21.2 \pm 6.3$ & $20.6 \pm 4.0$ & $19.0 \pm 4.4$ & $24.8 \pm 6.3$ & $26.6 \pm 9.9$ \\
Ankle Supination & $12.4 \pm 5.7$ & $16.6 \pm 5.5$ & $11.7 \pm 4.3$ & $14.9 \pm 7.3$ & $21.1 \pm 5.5$ & $20.8 \pm 7.8$ \\
\hline
\end{tabular}

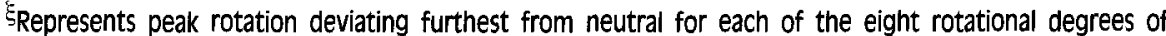
freedom. *Denotes statistically significant differences for the main effect of gender $(p<0.05)$.

EDenotes statistically significant differences between genders within a movement condition $(p<0.05)$.

Table 1: Effects of gender and movement condition on mean $( \pm S D)$ peak stance phase lower limb joint rotation variables.

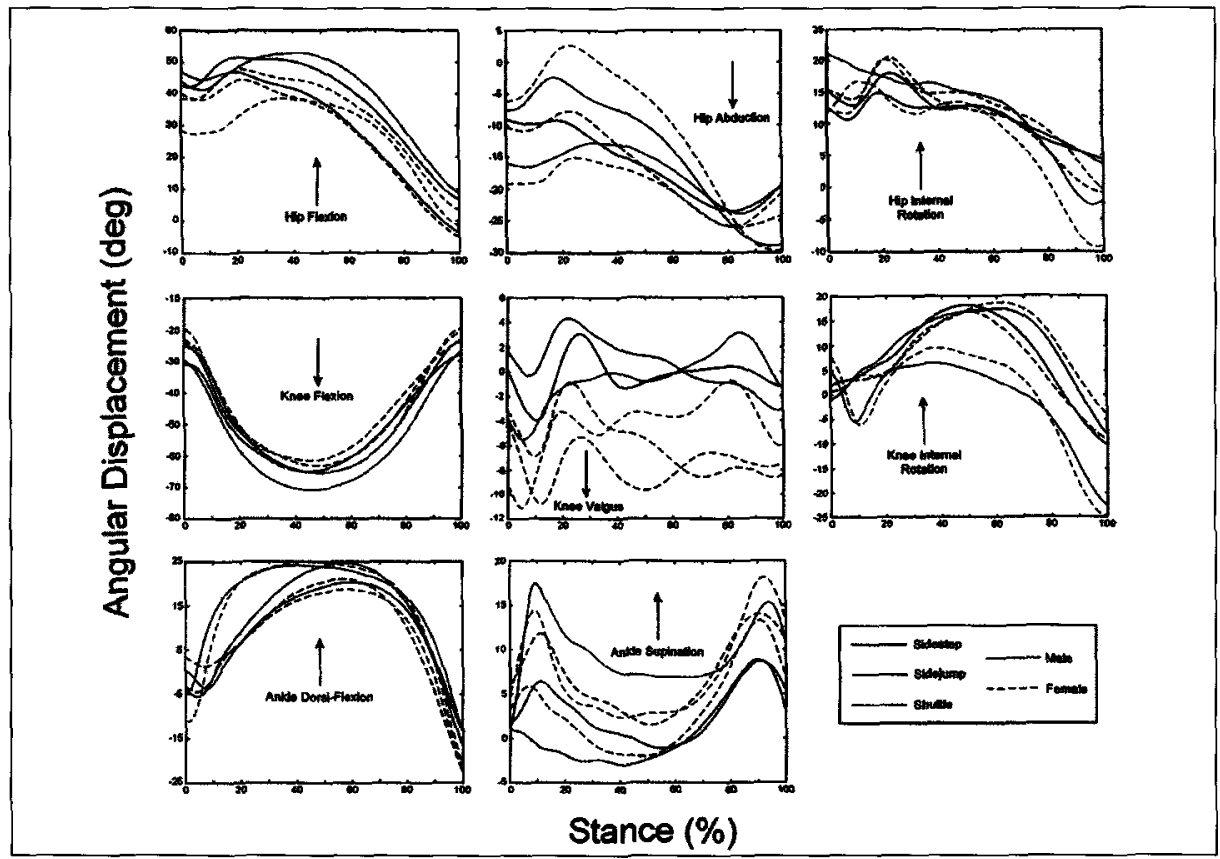

Figure 1: Effect of gender and movement condition (sidestep, sidejump and shuttle run) on threedimensional lower limb kinematic patterns during stance. Arrows indicate the sign convention for the joint angles. 


\begin{tabular}{|c|c|c|c|c|c|c|}
\hline \multirow[b]{2}{*}{ Rotation $\xi$ (degrees) } & \multicolumn{2}{|c|}{ Sidestep } & \multicolumn{2}{|c|}{ Jump-Land } & \multicolumn{2}{|c|}{ Shuttle Run } \\
\hline & Male & Female & Male & Female & Male & Female \\
\hline Hip Flex - Ext* & $46.9 \pm 8.5$ & $40.0 \pm 8.8$ & $42.5 \pm 10.0$ & $38.5 \pm 7.4$ & $44.0 \pm 10.7$ & $28.3 \pm 9.1 \xi$ \\
\hline Hip Abd - Add & $-9.0 \pm 7.1$ & $-10.1 \pm 4.8$ & $-7.6 \pm 4.6$ & $-6.2 \pm 5.5$ & $-15.9 \pm 5.5$ & $-19.2 \pm 6.5$ \\
\hline Hip I - E Rotn & $15.3 \pm 9.5$ & $15.3 \pm 10.5$ & $12.4 \pm 7.6$ & $12.0 \pm 10.8$ & $21.0 \pm 6.4$ & $11.6 \pm 6.9$ \\
\hline Knee Ext - Fle* & $\cdot 30.7 \pm 10.7$ & $-25.2 \pm 1.8$ & $-24.4 \pm 7.4$ & $-23.2 \pm 4.6$ & $-31.2 \pm 4.2$ & $-20.8 \pm 5.0^{\xi}$ \\
\hline Knee Var - Val* & $2.2 \pm 2.5$ & $-2.4 \pm 3.7 \xi$ & $3.7 \pm 2.3$ & $-1.1 \pm 2.6^{\xi}$ & $-1.8 \pm 1.6$ & $-7.3 \pm 3.2^{\xi}$ \\
\hline Knee I - E Rotn & $1.9 \pm 8.5$ & $2.0 \pm 8.6$ & $-1.1 \pm 6.9$ & $0.7 \pm 8.2$ & $4.2 \pm 8.7$ & $7.3 \pm 15.7$ \\
\hline Ankle D - P Flex & $-4.1 \pm 11.8$ & $-3.5 \pm 12.9$ & $0.4 \pm 12.6$ & $3.3 \pm 12.2$ & $-5.1 \pm 9.3$ & $-11.0 \pm 8.0$ \\
\hline Ankle Sup - Pro & $1.7 \pm 4.9$ & $6.3 \pm 5.6$ & $0.9 \pm 6.2$ & $3.4 \pm 9.0$ & $1.0 \pm 5.9$ & $5.3 \pm 5.4$ \\
\hline
\end{tabular}

Table 2: Effects of gender and movement condition on mean $( \pm S D)$ lower limb joint rotations at contact. Data convention is such that hip flexion, hip internal rotation, knee extension and ankle supination are denoted as positive.

\begin{tabular}{|c|c|c|c|c|c|c|}
\hline \multirow[b]{2}{*}{ Rotation( Idegrees) } & \multicolumn{2}{|c|}{ Sidestep } & \multicolumn{2}{|c|}{ Jump-Land } & \multicolumn{2}{|c|}{ Shuttle Run } \\
\hline & Male & Female & Male & Female & Male & Female \\
\hline Hip Flex - Ext & $2.6 \pm 0.4$ & $2.3 \pm 0.7$ & $2.8 \pm 0.5$ & $2.8 \pm 0.8$ & $4.1 \pm 1.5$ & $3.1 \pm 1.0$ \\
\hline Hip Abd - Add & $2.4 \pm 0.8$ & $2.1 \pm 0.6$ & $2.3 \pm 0.9$ & $2.3 \pm 0.5$ & $3.5 \pm 1.3$ & $2.7 \pm 0.7$ \\
\hline Hip I - E Rotn & $4.5 \pm 1.1$ & $4.5 \pm 1.0$ & $4.4 \pm 1.2$ & $3.9 \pm 1.5$ & $5.8 \pm 1.8$ & $6.4 \pm 2.2$ \\
\hline Knee Flex - Ext & $3.6 \pm 0.9$ & $3.0 \pm 1.1$ & $3.9 \pm 0.8$ & $3.8 \pm 1.7$ & $4.5 \pm 1.0$ & $4.0 \pm 1.3$ \\
\hline Knee Var - Val & $3.0 \pm 1.1$ & $2.9 \pm 0.8$ & $3.0 \pm 0.8$ & $3.0 \pm 1.3$ & $4.1 \pm 1.7$ & $2.9 \pm 0.8$ \\
\hline Knee I - E Rotn & $2.7 \pm 1.0$ & $2.6 \pm 0.8$ & $2.6 \pm 0.8$ & $2.5 \pm 0.6$ & $4.1 \pm 2.0$ & $3.6 \pm 1.1$ \\
\hline Ankle D - P Flex & $3.7 \pm 1.4$ & $3.4 \pm 0.7$ & $3.7 \pm 0.9$ & $4.2 \pm 1.2$ & $4.2 \pm 0.5$ & $4.5 \pm 1.6$ \\
\hline Ankle Sup - Pro & $1.8 \pm 0.5$ & $1.8 \pm 0.6$ & $1.9 \pm 0.6$ & $1.9 \pm 0.5$ & $2.4 \pm 0.8$ & $2.9 \pm 0.6$ \\
\hline
\end{tabular}

Table 3: Effects of gender and movement condition on mean ( \pm SD) stance phase lower limb joint rotation variability.

Gender differences were observed in three peak stance phase kinematic variables (Table 1). Specifically, females displayed significant increases in peak hip flexion $\left(\mathrm{p}=4 \times 10^{-3}\right)$, knee flexion $\left(\mathrm{p}=3 \times 10^{-2}\right)$ and knee valgus $\left(\mathrm{p}=1 \times 10^{-6}\right)$ compared to males (Figure 1). Post hoc analyses revealed that gender differences in peak hip flexion and knee flexion were evident only for the shuttle run movement condition. Gender differences in peak knee valgus, however, were evident within all three movements (Table 1). Significant interactions between gender and movement condition did not exist for any peak kinematic data comparisons.

Gender differences also existed in initial contact hip flexion-extension ( $p$ $\left.=4 \times 10^{-5}\right)$, knee flexion-extension $\left(\mathrm{p}=2 \times 10^{-3}\right)$ and knee varus-valgus $\left(\mathrm{p}=1 \times 10^{-6}\right)$ positions (Table 2). Specifically, females had significantly lower hip and knee flexion and greater knee valgus positions at contact compared to males. Gender differences in initial hip and knee flexion positions were again significant within the shuttle run movement condition only, while a gender difference in 


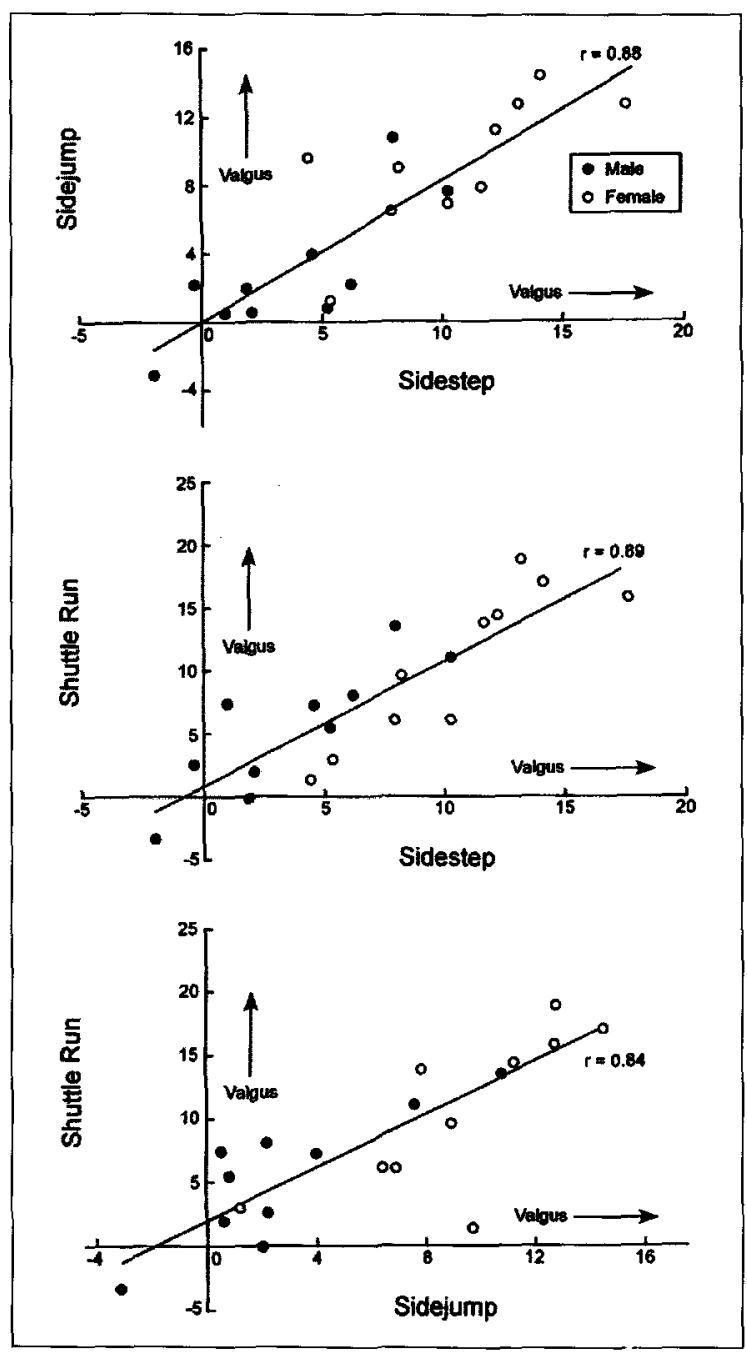

Figure 2: Correlations of subjectbased mean peak valgus measures between sidestep, sidejump and shuttle run movement conditions.

initial valgus position existed in all three movements. As with peak data measures, no significant interactions between gender and movement conditions were observed for initial contact data comparisons. Movement variability was not different between males and females (Table 3), and no significant interactions between gender and movement conditions existed for these results (Table 3).

Peak knee valgus angle was highly correlated between all three movements (Figure 2), with comparisons of sidestep and sidejump, sidestep and shuttle and sidejump and shuttle yielding correlation coefficients ( $r$ ) of $0.88,0.89$ and 0.84 respectively. 


\section{Discussion}

The current study aimed to determine if gender differences evident in 3D lower limb kinematics were consistent across several known high risk sporting postures. A number of studies have assessed gender differences in knee joint kinematics associated with isolated movement tasks, such as jump landing $8,13,15$ and cutting and pivoting movements ${ }^{9,10,17}$. Our data are consistent with these studies, both in terms of the overall movement patterns observed for these two movements and the associated rotational magnitudes. This appears to be the first time, however, that gender-based kinematic data have been compared for the entire lower limb across several sports movements in a single population. This approach is crucial to determining the transferability of potential gender differences, and thus the effective screening and modification of these differences in the future. This is also the first instance where gender-differences in lower limb motions have been evaluated for shuttle-run tasks. Inclusion of this movement was deemed important considering it combines the deceleration component of jump-landing with the rapid change in direction associated with pivoting tasks, which are both deemed hazardous in terms of ACL injury ${ }^{1,4}$.

Previous studies have shown individually that women demonstrate larger knee valgus angles than men during sidestep and ${ }^{9,10,17}$ and jump-landing tasks $^{8}$. In the current study, females landed with more knee valgus and demonstrated greater peak valgus compared to males for all three movements tested, with peak valgus data strongly correlated between these conditions. Relatively large knee valgus motions may thus be a common female trait across all sports movements linked to ACL injury. It also appears possible to identify individuals within each gender who consistently elicit high valgus angles, regardless of the movement task (see Figure 2).

With joint kinematics being examined for normal movement execution only, it is difficult to make strong inferences regarding causal links to ACL injury. Considering, however, that females also demonstrate an increased risk of sports-related ACL injury compared to males ${ }^{1}$, it may be that valgus motion is an important component of the underlying female injury mechanism. The recent work of Hewett et $\mathrm{al}^{21}$ supports this theory, showing prospectively that knee valgus during drop-jump execution predicts ACL injury risk in young female athletes. Further support is provided by computational models that simulate randomly-perturbed sidestepping movements, showing that valgus loads can easily rise to injurious levels during realistic movement control variations $^{18}$. If stance phase knee valgus is in fact a generalisable predictor of non-contact ACL injury risk, more simple and time-effective screening programs may be possible to detect abnormal valgus motions specifically. Prospective examinations of ACL injury risk and lower limb biomechanics for a wider range of movements would provide further insight here and thus appear a worthwhile research endeavour.

While individuals may demonstrate similar valgus motions across movements, it cannot automatically be assumed that this is due to a common underlying neuromuscular mechanism. Anatomical factors such as for example $\mathrm{G}$ angle or valgus alignment, which are known to demonstrate a degree of gender dimorphism, have been proposed as contributors to the 


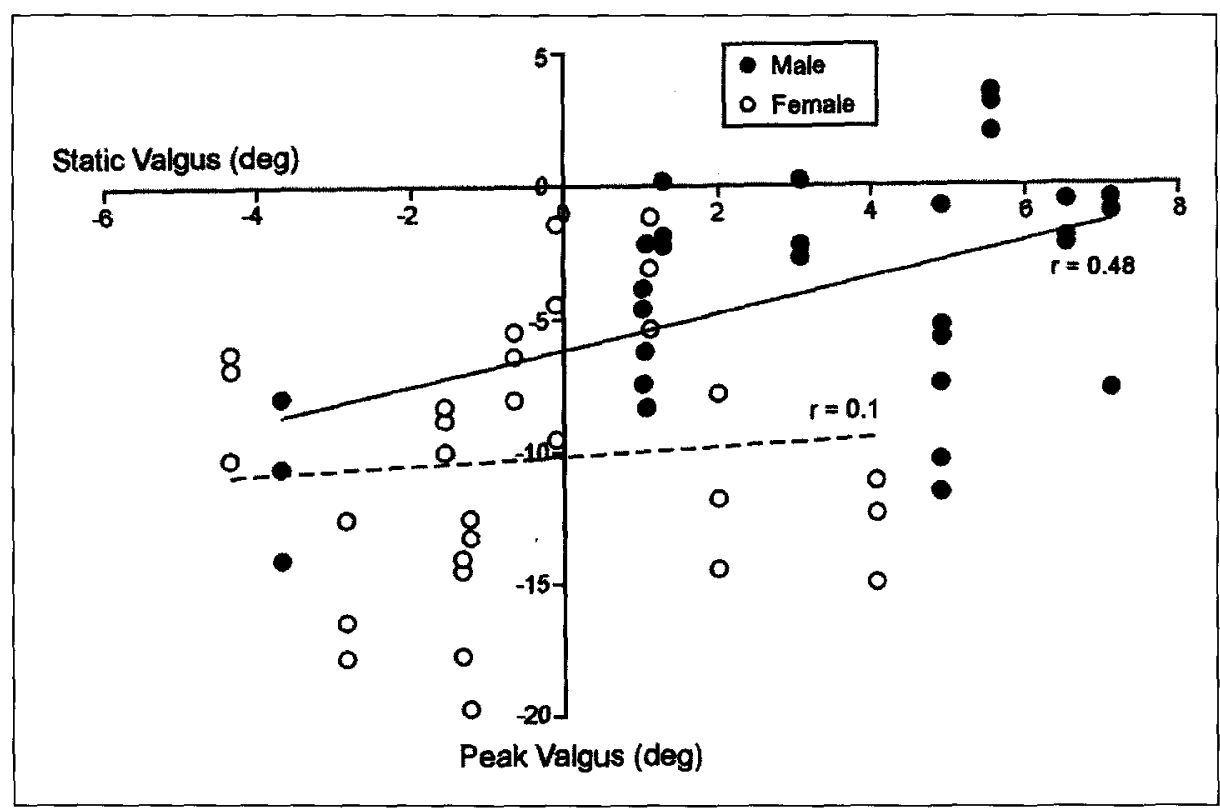

Figure 3: Correlation between static (neutral) knee valgus angle and peak valgus angle demonstrated during the stance phase of three sports movements for males ( $r=0.48, N=10$ subjects $x 3$ movements) and females ( $r=0.1, N=10$ subjects $\times 3$ movements).

increased valgus motions demonstrated by females ${ }^{4,22}$. We explored this possibility by computing static valgus from the coronal plane projections of hip, knee and ankle joint centres. We found that there was no significant correlation between peak stance phase valgus during movement tasks and the static valgus alignment of the subject (Figure 3). Knee laxity should also be considered as another factor common to all movements that may influence peak valgus ${ }^{23}$. We did not collect laxity data on the current test population, and this should be considered in further research. Considering, however, that within-subject, between-trial variations in knee valgus were of similar magnitude as the between-subject variations (see Tables 2,3), and that withinsubject changes in anatomy and laxity do not exist, current results strongly suggest that neuromuscular control plays an important role in resultant knee valgus. Regardless, the future success of ACL injury prevention strategies may lie in the ability to determine how anatomy, laxity and neuromuscular control interact to produce knee valgus, and ultimately, risk of ACL injury.

Females have been shown previously in some studies to land in a more extended position during sidestepping ${ }^{9,10}$ and jump landing tasks ${ }^{15,16}$. It has also been suggested that landing in this position increases the risk of ACL injury in women because of increased anterior tibial shear loads via the resultant action of the quadriceps ${ }^{9,11,24}$. In the current study, however, females only landed in a more extended position and demonstrated smaller flexion values during shuttle run movements. This observation suggests that gender differ- 
ences in hip and knee sagittal plane motions may be dependent both on the population and task/s being tested. This likely also explains why some studies have found females actually elicit increased knee flexion compared to males during similar movements ${ }^{17,25}$. Thus, if landing in a more extended position increases the risk of ACL injury, it does not appear to be a common female trait across sports movements. Screening of hip and knee flexion-extension patterns as a potential ACL injury mechanism therefore should extend beyond assessment of an isolated movement task.

Increased between-trial variability in sports movement execution has been suggested to increase the likelihood of performing a movement that precipitates high-risk joint postures ${ }^{14,17}$. Between-trial variability data for the hip, knee and ankle in the current case were not different between males and females for any of the movements tested. This observation contradicts our earlier work in which females had increased between-trial variations in out-of-plane knee rotations compared to males during sidestepping tasks ${ }^{10.17}$. Unlike those studies, current data were obtained for experience matched elite level athletes only and hence it may be, as suggested by McLean et al ${ }^{17}$, that movement variability is largely dependent on skill/experience level. Thus, at least for highly skilled basketball players, gender differences in sports-related ACL injury rates do not appear to stem from variability in movement performance. Movement variability cannot be discounted, however, as a contributing factor to the gender disparity in injury rates for other, less skilled populations. Exposing individuals and particularly females within such populations to increased movement specific drills as a means of reducing movement variability may still therefore be warranted.

\section{Potential Limitations}

There is recent evidence to suggest that hormonal fluctuations occurring throughout the menstrual phase may influence both knee joint laxity and muscle stiffness measures in females ${ }^{23,26}$. It is possible that these fluctuations, manifesting in resultant neuromuscular control, may in turn promote similar variations in resultant joint motions. To date, data on such effects are not available. We did not currently account for menstrual cycle phase and hence it is possible that female data may have been confounded by these pre-mentioned effects. If hormonal fluctuations did indeed influence female movement responses, and they were tested at random times within their menstrual cycle, one would expect relatively large inter-subject variations in kinematic data. Female group standard deviation data, however, were typically the same, and in some cases smaller, than corresponding male data (see Tables 1,2 and 3). Regardless, the potential contributions of hormonal fluctuations associated with the menstrual cycle to lower limb motions and loads during high-risk movements should be considered in future studies, particularly those focusing within rather than across gender.

A well known problem in calculating accu'rate knee joint kinematics is that valgus and internal rotation are small relative to the flexion-extension motion and therefore easily influenced by minor variations in the definition of the joint coordinate system ${ }^{27,28}$. This potential for "kinematic cross-talk" may have thus influenced both the reliability and repeatability of out-of-plane rotational data. The critical part of the analysis is the definition of the first (flexion) axis in the 
knee joint coordinate system. We currently define our knee flexion axis to be aligned with the global (lab) axis during standing, rather than through the epicondylar markers, to exclude variations due to erroneous identification of these landmarks. During the standing reference pose, we align the subject's pelvis and feet with the laboratory coordinate system. This knee joint coordinate system has been used in our recent study that identified knee valgus as a prospective predictor of ACL injury ${ }^{21}$. This suggests that the resulting valgus measures are reproducible and useful. Whether this variable represents the "true" anatomical valgus can be debated. In any marker-based study of 3-D joint motion extremity kinematics, the definition of the joint coordinate system and the potential for kinematic crosstalk must be carefully considered when interpreting the results.

\section{Conclusions}

Based on the research outcomes obtained for the population tested, the following conclusions can be drawn:

1. Females had increased initial knee valgus and peak knee valgus, when compared to males, in three sports movements linked to non-contact ACL injury.

2. Peak valgus is highly correlated between the three movements, which supports the validity of using this variable as a basis for ACL risk screening and neuromuscular training.

3. Gender differences in hip and knee flexion-extension excursions appear dependent on both the task and population under investigation.

4. In college level basketball players, there is no gender difference in the variability of lower limb movement.

\section{Acknowledgements}

This work was supported by U.S. National Institutes of Health (grant 1 R0l AR47039). We would also like to thank Dr Liang Li PhD from the Department of Biostatistics, The Cleveland Clinic Foundation, for his help with the statistical interpretation and analyses.

\section{References}

1. Arendt E, Dick R. Knee injury patterns among men and women in collegiate basketball and soccer. NCAA data and review of literature. Am J Sports Med, 1995, 23(6):694-701.

2. Feagin JA Jr, Lambert KL. Mechanism of injury and pathology of anterior cruciate ligament injuries. Orthop Clin North Am, 1985, 16(1):41-45.

3. Maletius W, Messner K. Eighteen- to twenty-four-year follow-up after complete rupture of the anterior cruciate ligament. Am J Sports Med, 1999, 27(6):711-717

4. Griffin LY, Agel J, Albohm MJ et al. Noncontact anterior cruciate ligament injuries: risk factors and prevention strategies. J Am Acad Orthop Surg, 2000, 8(3):141-150.

5 . Hewett TE, Stroupe AL, Nance TA et al. Plyometric training in female athletes. Decreased impact forces and increased hamstring torques. Am J Sports Med, 1996, 24(6):765-773.

6. Hewett TE, Lindenfeld TN, Riccobene JV et al. The effect of neuromuscular training on the incidence of knee injury in female athletes. A prospective study. Am J Sports Med. 1999, 27(6):699-706.

7. Myer GD, Ford KR, Palumbo JP et al. Neuromuscular training improves performance and lower-extremity biomechanics in female athletes. $J$ Strength Cond Res 2005, 19(1):51-60.

8. Ford KR, Myer GD, Hewett TE. Valgus knee motion during landing in high school female and male basketball players. Med Sci Sports Exerc, 2003, 35(10):1745-1750.

9. Malinzak RA, Colby SM, Kirkendall DT et al. A comparison of knee joint motion patterns 
between men and women in selected athletic tasks. Clin Biomech, 2001, 16(5):438-445.

10. McLean SG, Lipfert SW, Van Den Bogert AJ. Effect of Gender and Defensive Opponent on the Biomechanics of Sidestep Cutting. Med Sci Sports Exerc, 2004, 36(6): 1008-1016.

11. Colby S, Francisco A, Yu B et al. Electromyographic and kinematic analysis of cutting maneuvers. Implications for anterior cruciate ligament injury. Am J Sports Med, 2000, 28(2):234-240.

12. Zeller BL, McCrory JL, Kibler WB et al. Differences in kinematics and electromyographic activity between men and women during the single-legged squat. Am J Sports Med, 2003, 31(3):449-456.

13. Ferber R, Davis IM,Williams DS 3rd. Gender differences in lower extremity mechanics during running. Clin Biomech, 2003, 18(4):350-357.

14. Hurd WJ, Chmielewski TL, Axe MJ et al. Differences in normal and perturbed walking kinematics between male and female athletes. Clin Biomech, 2004, 19(5):465-472.

15. Decker MJ, Torry MR, Wyland DJ et al. Gender differences in lower extremity kinematics, kinetics and energy absorption during landing. Clin Biomech, 2003, 18(7):662-669.

16. Salci $\mathrm{Y}$, Kentel BB, Heycan $\mathrm{C}$ et al. Comparison of landing maneuvers between male and female college volleyball players. Clin Biomech, 2004, 19(6):622-628.

17. McLean SG, Neal RJ, Myers PT et al. Knee joint kinematics during the sidestep cutting maneuver: potential for injury in women. Med Sci Sports Exerc, 1999, 31(7):959-968.

18. McLean SG, Huang X, Su A et al. Sagittal plane biomechanics cannot injure the ACL during sidestep cutting. Clin Biomech, 2004, 19(8):828-838.

19. Wu G, Siegler S, Allard P et al. ISB recommendation on definitions of joint coordinate system of various joints for the reporting of human joint motion-part I: ankle, hip, and spine. International Society of Biomechanics. J Biomech, 2002, 35(4):543-548.

20. Woltring HJ. A Fortran package for generalized, cross-validatory spline smoothing and differentiation. Adv Eng Software, 1986, 8:104-107.

21. Hewett TE, Myer GD, Ford KR et al. Biomechanical measures of neuromuscular control and valgus loading of the knee predict anterior cruciate ligament injury risk in female athletes: a prospective study. Am J Sports Med 2005, 33(4):492-501.

22. Loudon JK, Jenkins $W$, Loudon KL. The relationship between static posture and ACL injury in female athletes. J Orthop Sports Phys Ther, 1996, 24(2):91-97.

23. Wojtys EM, Huston LJ, Schock HJ et al. Gender differences in muscular protection of the knee in torsion in size-matched athletes. $J$ Bone Joint Surg Am, 2003, 85-A:782-789.

24. Chappell JD, Yu B, Kirkendall DT et al. A comparison of knee kinetics between male and female recreational athletes in stop-jump tasks. Am $J$ Sports Med, 2002, 30(2):261-267.

25. Fagenbaum R. Darling WG. Jump landing strategies in male and female college athletes and the implications of such strategies for anterior cruciate ligament injury. Am $J$ Sports Med, 2003, 31(2):233-240.

26. Shultz SJ, Kirk SE, Johnson ML et al. Relationship between sex hormones and anterior knee laxity across the menstrual cycle. Med Sci Sports Exerc 2004, 36(7):1165-1174.

27. Piazza SJ, Cavanagh PR. Measurement of the screw-home motion of the knee is sensitive to errors in axis alignment. $J$ Biomech, 2000; 33(8): 1029-1034.

28. Ramakrishnan HK, Kadaba MP. On the estimation of joint kinematics during gait. $J$ Biomech 1991, 24(10):969-977. 